\title{
Compliance in oxygen saturation targeting in preterm infants: a systematic review
}

\author{
Henriëtte A. van Zanten ${ }^{1} \cdot$ Ratna N. G. B. Tan ${ }^{1}$ • Agnes van den Hoogen ${ }^{2}$ • \\ Enrico Lopriore $^{1} \cdot$ Arjan B. te Pas ${ }^{1}$
}

Received: 11 July 2015 /Revised: 24 September 2015 / Accepted: 28 September 2015 / Published online: 14 October 2015

(C) The Author(s) 2015. This article is published with open access at Springerlink.com

\begin{abstract}
During oxygen therapy in preterm infants, targeting oxygen saturation is important for avoiding hypoxaemia and hyperoxaemia, but this can be very difficult and challenging for neonatal nurses. We systematically reviewed the qualitative and quantitative studies investigating the compliance in targeting oxygen saturation in preterm infants and factors that influence this compliance. We searched PubMed, Embase, Web of Science, Cochrane, CINAHL and ScienceDirect from 2000 to January 2015. Sixteen studies were selected, which involved a total of 2935 nurses and 574 infants. The studies varied in methodology, and we have therefore used a narrative account to describe the data. The main finding is that there is a low compliance in oxygen targeting; the upper alarm limits are inappropriately set, and maintaining the saturation $\left(\mathrm{SpO}_{2}\right)$ below the upper limit presented particular difficulties. Although there is little data available, the studies indicate that training, titration protocols and decreasing workload could improve awareness and compliance. Automated oxygen
\end{abstract}

Communicated by Patrick Van Reempts

Henriëtte A. van Zanten

h.a.van_zanten@lumc.nl

Ratna N. G. B. Tan

r.n.g.b.tan@1umc.nl

Agnes van den Hoogen

ahoogen@lumc.nl

Enrico Lopriore

e.lopriore@lumc.nl

Arjan B. te Pas

a.b.te_pas@lumc.nl

1 Department of Pediatrics, Division of Neonatology, Leiden University Medical Center, J6-S, PO Box 9600, 2300 RC Leiden, The Netherlands

2 Utrecht Medical Center, Utrecht, The Netherlands regulations have been shown to increase the time that $\mathrm{SpO}_{2}$ is within the target range.

Conclusion: The compliance in targeting oxygen during oxygen therapy in preterm infants is low, especially in maintaining the $\mathrm{SpO}_{2}$ below the upper limit.

What is Known:

- The use of oxygen in preterm infants is vital, but the optimal strategy remains controversial.

- Targeting $\mathrm{SpO}_{2}$ during oxygen therapy in preterm infants has been shown to reduce mortality and morbidity.

What is New:

- Review of the literature showed that the compliance in targeting $\mathrm{SpO}_{2}$ and alarm settings is low.

- Creating awareness of risks of oxygen therapy and benefits in targeting, decreasing nurse/patient ratio and automated oxygen therapy could increase compliance.

Keywords Preterm infant - Targeting oxygen - Compliance . Alarm limits $\cdot$ Hyperoxaemia $\cdot$ Hypoxaemia $\cdot$ Automated oxygen

$\begin{array}{ll}\text { Abbreviations } \\ \text { CPAP } & \text { Continuous positive airway pressure } \\ \text { NICU } & \text { Neonatal intensive care unit } \\ \mathrm{PO} & \text { Pulse oximetry } \\ \mathrm{SpO}_{2} & \text { Oxygen saturation } \\ \mathrm{TRs} & \text { Target ranges }\end{array}$

\section{Introduction}

Supplemental oxygen is often administered to preterm infants for hypoxemic episodes during respiratory distress or 
apnoeas. It is important to prevent hypoxaemia (defined as a decrease in arterial blood saturation $\left(\mathrm{SpO}_{2}\right)$ of $\leq 80 \%$ for $\geq 10 \mathrm{~s}$ ), as frequent episodes could lead to an increased risk of morbidities, including retinopathy of prematurity (ROP), impaired growth, longer term cardio-respiratory instability and adverse neurodevelopmental outcome [12, 15, $30]$. In extreme cases, it can even lead to death $[12,15]$. Hyperoxaemia $\left(\mathrm{SpO}_{2}\right.$ of $>95 \%$ for $\geq 10$ s) also needs to be prevented, as administering supplemental oxygen can potentially lead to high oxygen levels. High concentration of oxygen is toxic to living cells and is known to be an important pathogenic factor for bronchopulmonary dysplasia (BPD) and ROP [31] and is correlated with cerebral palsy [3].

Pulse oximetry (PO) is most commonly used for continuous monitoring of oxygen saturation $\left(\mathrm{SpO}_{2}\right)$ in a noninvasive manner [26]. To prevent hypoxaemia and hyperoxaemia, nurses usually titrate oxygen manually to maintain $\mathrm{SpO}_{2}$ between the prescribed target ranges. However, maintaining the $\mathrm{SpO}_{2}$ within this range can be challenging, and compliance - defined as the nurse's behaviour that follows the clinical guidelines-[13] is influenced by several factors [40]. This compliance is important, as it can largely influence the effect of a certain $\mathrm{SpO}_{2}$ target range. The optimal range of $\mathrm{SpO}_{2}$ for preterm infants remains undefined, but recent trials have shown that aiming for $91-95 \%$ has decreased mortality but increased incidence of ROP [36]. However, in these trials, oxygen was titrated manually, which caused a large overlap in the distribution of $\mathrm{SpO}_{2}$ between the two groups and may have decreased the observed differences in outcome.

Although comparison of $\mathrm{SpO}_{2}$ target ranges has been subject to systemic review [19], a review in the compliance in $\mathrm{SpO}_{2}$ target ranges is not available but equally important as which target range is optimal. The purpose of this study is to systematically review the available literature in complianceand the factors influencing this compliance-in targeting $\mathrm{SpO}_{2}$ in preterm infants.

\section{Methods}

We performed a systematic review, following PRISMA guidelines where possible (Fig. 1) [28]. The aim of the PRISMA statement is to help authors improve the reporting of systematic reviews and meta-analyses, which made it a particularly useful framework for this report. Eligible studies were identified by searching online databases from January 1965 to January 2015 in PubMed, Embase, Web of Science, Cochrane, CINAHL and ScienceDirect (keywords in Table 1). After selecting the eligible studies, we manually searched the reference lists of the selected studies to identify additional references.
The criteria for inclusion limited the selection to articles published in English or Dutch which referred to preterm infants, (nursing) compliance, $\mathrm{SpO}_{2}$ monitoring by $\mathrm{PO}$ and targeting oxygen saturation during NICU admission. Both qualitative and quantitative designs were included, but publications that were not primary research studies, i.e. letters, abstracts, reviews and editorials, were not (Fig. 1).

Three authors (HvZ, RT, AH) independently graded the selected studies using the QualSyst tool for quantitative and qualitative studies [21]. In case of disagreement, consensus was reached through discussion or consultation of a fourth co-author (AtP). The QualSyst tool for quantitative studies is a validated generic checklist consisting 14 items with scores from zero to two and the possibility to score 'not applicable'. Items rated not applicable were excluded from the calculation of the summary score. The maximum total score is 28 . The summary score was calculated by summing the total score obtained across the relevant items and dividing that by the total possible score.

The QualSyst tool for qualitative studies is a validated generic checklist consisting of ten items with scores from zero to two, with the maximum total score of 20. A summary score was calculated for each study by summing the total score across the ten items and dividing them by the total possible score of 20 [21].

Data from selected studies were extracted using a data extraction form. The following study characteristics were extracted: author, year, design, sample, time points, length of measurement, target range and key results.

\section{Results}

Sixteen articles met the inclusion criteria for this review (Fig. 1), detailing studies that included a total of 574 infants and 2935 nurses. Fourteen of these studies used a quantitative design $[1,7-10,17,18,22,25,27,34,38,39,41]$ while the remaining two used qualitative methods $[2,29]$. There was no homogeneity in the study designs, so pooling the data for meta-analysis was not possible. We therefore discuss the studies and their results using a narrative format organized under thematic headings and summarized in tables.

\section{Quality assessment}

The studies varied in quality, but none was excluded because of low-quality scores. One observed weakness was the lack of power analysis in four of the studies [10, $17,27,39]$, and all studies were unclear in the reasoning behind the timing and duration of $\mathrm{SpO}_{2}$ data collection [1, 
Fig. 1 Flow diagram selection studies
2, 7, 10, 17, 18, 22, 25, 27, 29, 34, 38, 39, 41] (Tables 2 and 3 ).

\section{Study designs}

The designs of the quantitative studies varied and were composed of the following: one efficacy study [9], two pilot clinical trials [6, 41], three randomized clinical trials [7, 18, 38] and eight observational studies, of which six had a prospective design $[1,10,17,22,25,27]$ and two were retrospective [34, 39] (Table 4). Both qualitative studies employed a descriptive design $[2,29]$ (Table 4).

\section{TRs of $\mathrm{SpO}_{2}$}

The lower limit of the target ranges (TRs) varied between studies from 80 to $92 \%[17,18]$, and upper limits of TR varied from 92 to $96 \%$, respectively $[1,9,10,17,25,27$, 34] (Table 4).

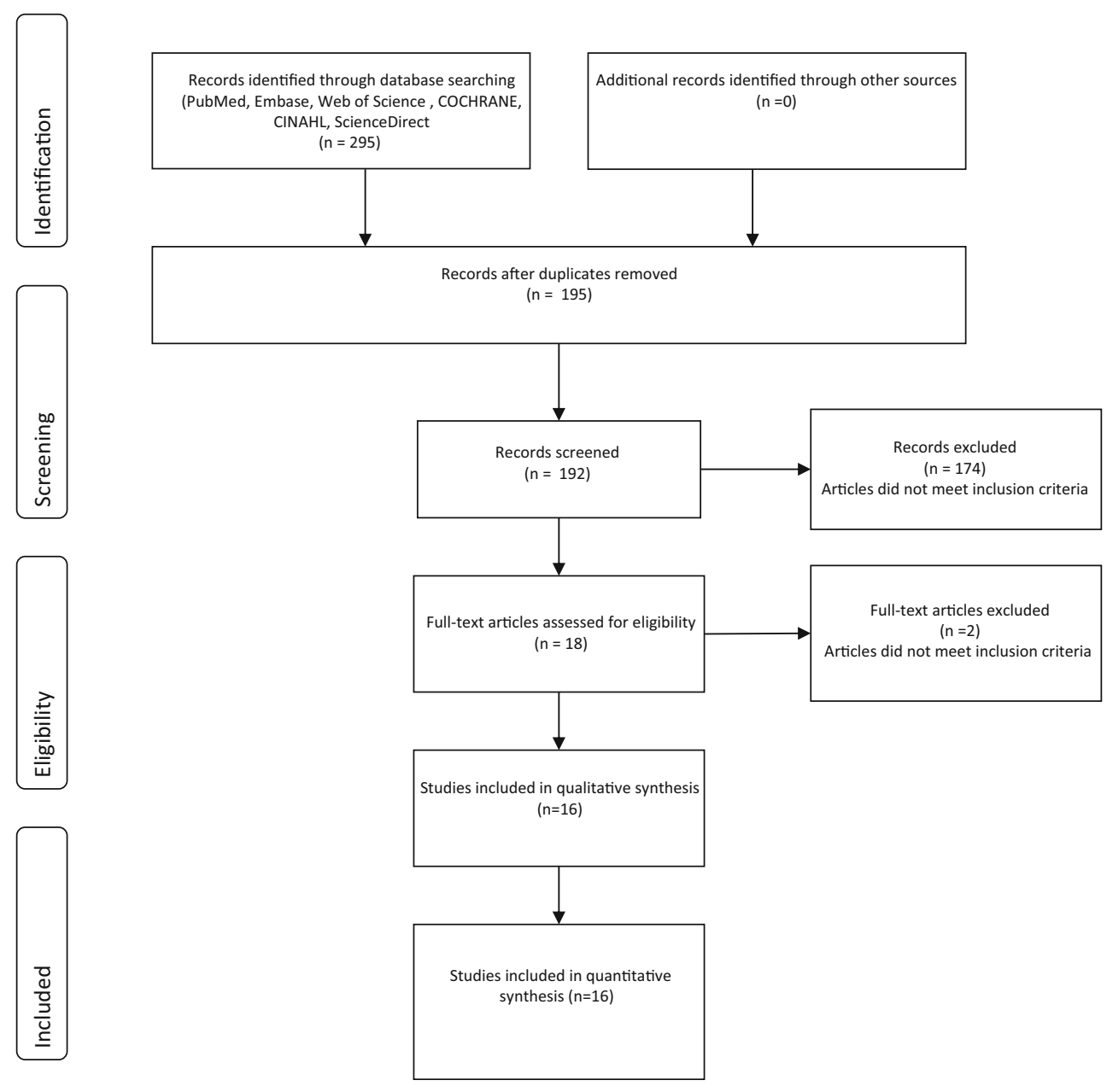

Time points and length of measurements

All studies were conducted in the period that the infants needed supplemental oxygen, but the starting time points and duration of data collection differed between studies. The starting time point varied between the first day of life [1, 2] and 33 days [8] (Table 4). In one study, the postnatal age was not described [27]. The duration of data collection also varied widely, the shortest covering only $4 \mathrm{~h}$ [9] and the longest the entire period between admission and discharge [10]. The data were collected continuously in eight studies [1, 7-9, 18, 34, 38, 39, 41] and intermittently in the remaining studies [10, 17, 22, 27] (Table 4).

\section{Compliance in TR}

Twelve studies investigated how often $\mathrm{SpO}_{2}$ values were in or outside the TR, expressed as the percentage of monitored time $[1,7-9,17,18,22,25,34,38,39,41]$. In a multicentre study, Hagadorn et al. observed that $\mathrm{SpO}_{2}$ was below, within or above TR in $16(0-47 \%), 48(6-75 \%)$ and $36(5-90 \%)$, respectively, of the monitored time [17]. Van der Eijk et al. reported similar 
Table 1 Keywords in different databases

Database Keywords (including MeSH) terms

PubMed

Hyperoxia ${ }^{a}$, Hyperoxia*, hyperoxygenation, Hyperoxias, Hyperoxie, Hyperoxic, Hyperox*, hyperoxemic episode, hyperoxemic episodes, hypoxia, hypox*, hypoxemic episode, hypoxemic episodes, cyanosis cyanoses pulse oximetry, pulse oximeter, pulse oximeters, Infant ${ }^{\mathrm{a}}$, Premature ${ }^{\mathrm{a}}$, prematurity, prematur*, Pre-mature, pre-maturity, preterm, preterm*, low birth weight infant, low birth weight infants, Oxygen Inhalation Therapy ${ }^{\mathrm{a}}$, Hyperbaric Oxygenation ${ }^{\mathrm{a}}$, Oxygen/administration and dosage $\mathrm{a}^{\mathrm{a}}$, oxygen/therapeutic use + Oxygen/therapy ${ }^{\mathrm{a}}$, Oxygen/Consumption ${ }^{\mathrm{a}}$, oxygen consumption, oxygen, oxygenation, $\mathrm{FiO} 2, \mathrm{FiO} 2$, $\mathrm{FiO}(2), \mathrm{FiO}$, increas*, fraction* exposure*, increase oxygen, increased oxygen, oxygen supplementation, oxygen therapy, supplemental oxygen, Automated closed loop control, FIO2 automatic, FIO2 adjustment closed-loop, FIO2 control, Oxygen Inhalation Therapy/instrumentation ${ }^{\text {a }}$, Respiration, Artificial/instrumentation $^{\text {a }}$

complia*, nursing compliance, Adherence, adher*, Guideline Adherence ${ }^{\mathrm{a}}$, Advance Directive Adherence ${ }^{\mathrm{a}}$, Goals ${ }^{\mathrm{a}}$, nursing procedures

CINAHL Hyperoxia, hyperoxias, Hyperoxia* hyperoxygenation, hyperoxie, hyperoxic, hyperox* cyanosis, cyanoses, hypoxia*, pulse oximetry, pulse oximeter, pulse oximeters, prematur*, Prematurity, pre-mature, pre-maturity, preterm, preterm*, pre-term, low birth weight infant, low birth weight infants, Oxygen*, $\mathrm{FiO} 2, \mathrm{FiO} 2, \mathrm{FiO}(2), \mathrm{FiO}$, increas*, fraction, fractions, fraction*, exposure, exposures, exposure*, increase oxygen, increased oxygen, oxygen supplementation, supplemental oxygen, oxygen saturation, oxygen administration, oxygen therapy, Automated closed loop control, FIO2 automatic, FIO2 adjustment closed-loop, FIO2 control, compliance, complia*, nursing compliance, Adherence, adher*

Web of Science

Hyperoxia, Hyperoxias, Hyperoxia*, Hyperoxie, Hyperoxic, hyperoxygenation, Hyperox*, cyanosis, cyanoses pulse oximetry, pulse oximeter, pulse oximeters, hypoxia*, hypoxemic episodes, hyperoxemic episodes, hypoxemic episode, hyperoxemic episode, cyanosis, cyanoses, premature, Prematurity, prematur*, pre-mature, pre-maturity, preterm, preterm*, pre-term, elbw infant*, low birth weight infant*, Oxygen*, FiO2, $\mathrm{FiO} 2, \mathrm{FiO}(2), \mathrm{FiO}$, increas*, fraction, fractions, fraction*, exposure, exposures, exposure*, increase oxygen, increased oxygen, oxygen supplementation, supplemental oxygen, oxygen saturation, oxygen administration, oxygen therapy, Automated closed loop control, FIO2 automatic, FIO2 adjustment closed-loop, FIO2 control, compliance, complia*, nursing compliance, Adherence OR adher*

Embase

Hyperoxia/, pulse oximetry/, exp Hypoxia/, Hyperoxia, Hyperoxias, Hyperoxia*, Hyperoxie, Hyperoxic, hyperoxygenation, Hyperox*, pulse oximetry, pulse oximeter, pulse oximeters, hypoxia, hypoxemic episodes, "hyperoxemic episodes" hypoxemic episode, hyperoxemic episode, cyanosis/, cyanosis, cyanoses, prematurity/, premature, Prematurity, prematur*, pre-mature, pre-maturity, preterm, preterm*, pre-term, low birth weight infant, low birth weight infants, Oxygen*, FiO2, $\mathrm{FiO} 2$, $\mathrm{FiO}(2), \mathrm{FiO})$ increas*, fraction, fractions, fraction*, exposure, exposures, exposure*, increase oxygen, increased oxygen, oxygen supplementation, supplemental oxygen, oxygen saturation, oxygen administration, oxygen therapy, exp oxygen therapy/, oxygen saturation/, Automated closed loop control, FIO2 automatic, FIO2 adjustment closed-loop, FIO2 control, oxygen delivery device/, exp *patient compliance/ compliance, complia*, nursing compliance, Adherence, adher* "nursing procedures"

ScienceDirect

Hyperoxia, Hyperoxias, Hyperoxia*, Hyperoxie, Hyperoxic, hyperoxygenation, Hyperox*, pulse oximetry, pulse oximeter, pulse oximeters, hypoxia*, cyanosis, cyanoses, premature, Prematurity, prematur*, pre-mature, pre-maturity, preterm, preterm*, pre-term, low birth weight infant, low birth weight infants, Oxygen*, FiO2, $\mathrm{FiO} 2, \mathrm{FiO}(2), \mathrm{FiO}$, increas*, fraction, fractions, fraction*, exposure, exposures, exposure*, increase oxygen, increased oxygen, oxygen supplementation, supplemental oxygen, oxygen saturation, oxygen administration, oxygen therapy, Automated closed loop control, FIO2 automatic, FIO2 adjustment closed-loop, FIO2 control, compliance OR complia* OR nursing compliance OR Adherence OR adher*

Cochrane

Hyperoxia, Hyperoxias, Hyperoxia*, Hyperoxie, Hyperoxic, hyperoxygenation, Hyperox*, pulse oximetry, pulse oximeter, pulse oximeters, hypoxia*, premature, Prematurity, prematur*, pre-mature, pre-maturity, preterm, preterm*, pre-term, low birth weight infant, low birth weight infants, Oxygen*, FiO2, $\mathrm{FiO} 2$, $\mathrm{FiO}(2)$, FiO, increas*, fraction, fractions, fraction*, exposure, exposures, exposure*, increase oxygen, increased oxygen, oxygen supplementation, supplemental oxygen, oxygen saturation, oxygen administration, oxygen therapy,

Automated closed loop control, FIO2 automatic, FIO2 adjustment closed-loop, FIO2 control, compliance, complia*, nursing compliance, Adherence, adher*

\footnotetext{
${ }^{\mathrm{a}}$ Keywords that were MeSH terms
}

values, finding that $\mathrm{SpO}_{2}$ was below TR for $16 \%$ of the time and above it for $30 \%$ [39]. In contrast, Lim et al. only studied infants receiving supplemental oxygen during CPAP and $\mathrm{SpO}_{2}$ was below TR for $9 \%$ and above it for $58 \%$ of the time [25].

\section{Education and training}

Two studies demonstrated the impact of an educational program in targeting $\mathrm{SpO}_{2}$. Laptook et al. observed that training 
Table 2 Quality appraisal of included quantitative studies

\begin{tabular}{|c|c|c|c|c|c|c|c|c|c|c|c|c|}
\hline \multicolumn{13}{|c|}{ Quality assessment quantitative studies } \\
\hline Studies & Question & \multicolumn{2}{|c|}{$\begin{array}{l}2 . \\
\text { Study design }\end{array}$} & $\begin{array}{l}3 . \\
\text { Selection }\end{array}$ & \multicolumn{2}{|c|}{4.} & & \multicolumn{2}{|c|}{6.} & $\begin{array}{l}7 . \\
\text { Blinding subjects }\end{array}$ & $\begin{array}{l}8 . \\
\text { Outcome }\end{array}$ \\
\hline Claure, N. et al. (2001) & 1 & 1 & & 1 & 2 & & 1 & & 0 & & $\mathrm{n} / \mathrm{a}$ & 1 \\
\hline Claure, N. et al. (2009) & 1 & 1 & & 1 & 2 & & 1 & & 0 & & $\mathrm{n} / \mathrm{a}$ & 1 \\
\hline Claure, N. et al. (2011) & 2 & 2 & & 2 & 2 & & 1 & & 0 & & 0 & 2 \\
\hline Clucas, L. et al. (2007) & 2 & 2 & & 1 & 2 & & 0 & & 0 & & 0 & 2 \\
\hline Hagadorn, J.I. et al. 2006) & 2 & 2 & & 1 & 2 & & 1 & & 0 & & 0 & 1 \\
\hline Laptook, A.R. et al. (2006) & 1 & 1 & & 1 & 2 & & 0 & & 0 & & 0 & 1 \\
\hline Mills, B.A. et al. (2010) & 2 & 2 & & 1 & 2 & & 1 & & 0 & & 0 & 1 \\
\hline Sink, D.W. et al. (2011) & 2 & 1 & & 1 & 1 & & 0 & & 0 & & $\mathrm{n} / \mathrm{a}$ & 1 \\
\hline Urschitz, M.S. et al. (2004) & 2 & 2 & & 2 & 2 & & 2 & & 0 & & 0 & 1 \\
\hline Van der Eijk, A.C. et al. (2012) & 1 & 2 & & 2 & 2 & & 0 & & 0 & & 0 & 1 \\
\hline Zapata, J. et al. (2014) & 2 & 2 & & 2 & 2 & & 2 & & 0 & & 0 & 2 \\
\hline Lim, K. et al. (2014) & 2 & 2 & & 2 & 2 & & $\mathrm{n} / \mathrm{a}$ & & $\mathrm{n} / \mathrm{a}$ & & $\mathrm{n} / \mathrm{a}$ & 2 \\
\hline Arawiran, J. et al. (2014) & 2 & 2 & & 2 & 2 & & $\mathrm{n} / \mathrm{a}$ & & $\mathrm{n} / \mathrm{a}$ & & $\mathrm{n} / \mathrm{a}$ & 1 \\
\hline Hallenberger, A. et al. (2014) & 2 & 2 & & 2 & 2 & & 2 & & 0 & & 0 & 2 \\
\hline \multicolumn{13}{|c|}{ Quality assessment quantitative studies } \\
\hline Studies & \multicolumn{2}{|c|}{$\begin{array}{l}9 . \\
\text { Sample size }\end{array}$} & \multicolumn{3}{|c|}{$\begin{array}{l}10 . \\
\text { Analytic methods }\end{array}$} & \multicolumn{2}{|c|}{$\begin{array}{l}11 . \\
\text { Estimate of variance }\end{array}$} & \multicolumn{2}{|c|}{$\begin{array}{l}12 . \\
\text { Confounding }\end{array}$} & $\begin{array}{l}13 . \\
\text { Results }\end{array}$ & $\begin{array}{l}14 . \\
\text { Conclusion }\end{array}$ & $\begin{array}{l}\text { Summary } \\
\text { score }\end{array}$ \\
\hline Claure, N. et al. (2001) & \multicolumn{2}{|c|}{$\mathrm{n} / \mathrm{a}$} & \multicolumn{3}{|c|}{2} & \multirow{2}{*}{\multicolumn{2}{|c|}{$\begin{array}{l}2 \\
2\end{array}$}} & 1 & & 1 & 1 & $14 / 24=0.58$ \\
\hline Claure, N. et al. (2009) & \multicolumn{2}{|l|}{2} & \multicolumn{3}{|l|}{2} & & & \multicolumn{2}{|l|}{$\begin{array}{l}1 \\
1\end{array}$} & 1 & 1 & $16 / 26=0.62$ \\
\hline Claure, N. et al. (2011) & \multicolumn{2}{|l|}{2} & \multicolumn{3}{|l|}{2} & \multicolumn{2}{|l|}{2} & \multicolumn{2}{|l|}{1} & 2 & 2 & $22 / 28=0.79$ \\
\hline Clucas, L. et al. (2007) & 0 & & 2 & & & 2 & & 1 & & 2 & 2 & $18 / 28=0.64$ \\
\hline Hagadorn, J.I. et al. 2006) & 0 & & 2 & & & 2 & & 1 & & 1 & 1 & $16 / 28=0.57$ \\
\hline Laptook, A.R. et al. (2006) & 2 & & 2 & & & 2 & & 1 & & 1 & 1 & $15 / 28=0.54$ \\
\hline Mills, B.A. et al. (2010) & 0 & & 2 & & & 2 & & 1 & & 1 & 2 & $17 / 28=0.61$ \\
\hline Sink, D.W. et al. (2011) & $\mathrm{n} / \mathrm{a}$ & & 2 & & & 0 & & 1 & & 1 & 1 & $11 / 24=0.46$ \\
\hline Urschitz, M.S. et al. (2004) & 2 & & 2 & & & 2 & & 1 & & 2 & 2 & $22 / 28=0.79$ \\
\hline Van der Eijk, A.C. et al. (2012) & 0 & & 2 & & & 1 & & 1 & & 1 & 1 & $14 / 28=0.5$ \\
\hline Zapata, J. et al. (2014) & 1 & & 2 & & & 2 & & 1 & & 2 & 2 & $22 / 28=0.79$ \\
\hline Lim, K. et al. (2014) & $\mathrm{n} / \mathrm{a}$ & & 2 & & & 2 & & 2 & & 2 & 2 & $20 / 20=1$ \\
\hline Arawiran, J. et al. (2014) & 1 & & 2 & & & 2 & & 1 & & 2 & 1 & $18 / 22=0.82$ \\
\hline Hallenberger, A. et al. (2014) & 2 & & 2 & & & 2 & & 1 & & 1 & 1 & $21 / 28=0.75$ \\
\hline
\end{tabular}

$2=$ yes $; 1=$ partial $; 0=$ no; $\mathrm{n} / \mathrm{a}=$ not applicable

did not change the time that $\mathrm{SpO}_{2}$ was below (26.9 vs. $26.6 \%$; not significant (ns)) or above TR (15.4 vs. $14.0 \%$; ns) [22]. Interestingly, Arawiran et al. even observed that training had an adverse effect and that the time that $\mathrm{SpO}_{2}$ was within TR decreased after training $(44.5 \pm 14.4$ vs $40.4 \pm$ $12.8 \%$ ) with an increase in time above TR (from $36.9 \pm$ 17.2 vs $41.9 \pm 15.6 \%$ ) [1].

\section{Nurse/patient ratio}

Sink et al. studied the influence of the nurse/patient ratio on compliance in $\mathrm{SpO}_{2}$ targeting. They observed that the proportion of time that $\mathrm{SpO}_{2}$ was below TR decreased from 0.06 to 0.03 and time above TR increased from 0.56 to 0.82 when a third or fourth patient was added to the nurse's workload [34]. The high percentage of time above TR was probably due to the use of a lower upper limit (92\%) in comparison with other studies [7-9, 22, 38, 39]. Lim et al. also confirmed that more than one infant per nurse was associated with an increase in the time when $\mathrm{SpO}_{2}$ was above TR (Table 4) [25].

\section{Automated regulation of inspired oxygen}

Six recent studies reported that, when compared to manual titration, the use of automated regulation of inspired oxygen increased the time that $\mathrm{SpO}_{2}$ spent within TR [7-9, 18, 38, 41]. In a multicenter crossover study of ventilated preterm infants, Claure et al. (2011) observed that the time that $\mathrm{SpO}_{2}$ 
was within TR increased significantly during the automated period compared with the manual period (40\% (14) vs $32 \%$ (13) (mean (SD) $p<0.001$ ). The time periods with $\mathrm{SpO}_{2}$ $>93 \%$ or $>98 \%$ were thus significantly reduced during the automated period [7]. Although most studies observed that the time that $\mathrm{SpO}_{2}$ was above TR decreased [7-9, 38, 41] while the time below TR increased [7, 8, 38, 41], Hallenberger et al. found different results. They observed no change in time above TR (16 (0.0-60) vs $15.9(1.9-34.8) p=0.108)$ during automatic control of inspired oxygen and, therefore, no difference with manual control [18] (Table 4).

\section{Compliance in alarm limit setting}

Two studies investigated nursing compliance in setting the appropriate alarm limits for PO in preterm infants $[10,27]$. The actual $\mathrm{SpO}_{2}$ values were not reported, but Clucas et al. observed that the lower and upper alarm limit was set correctly in 91 and $23 \%$ of monitored time, respectively [10]. Mills et al. compared compliance in alarm settings of $\mathrm{SpO}_{2}$ according to whether or not infants participated in a trial. When infants were participating in the BOOST II trial, the lower and upper alarm limit for $\mathrm{SpO}_{2}$ was set correctly in $94 \%$ $(88-100 \%)$ and $80 \%(71-88 \%)$ of the monitored time period. However, this decreased to $87 \%$ (75-99\%) and $29 \%$ $(17-40 \%)$ when infants were not participating in the trial [27] (Table 4).

\section{Nurses' perception and awareness}

Armbruster et al. interviewed nurses who stated that the following would improve their compliance: further education, prompt response to alarm limits, a favourable patient to staff ratio, root cause analyses at the bedside and high priority given to control oxygen therapy [2]. Nghiem et al. reported that $63 \%$ of the nurses were aware of the local oxygen saturation guidelines and $57 \%$ of them correctly identified the target limits specified by their NICU guidelines (Table 4) [29].

\section{Discussion}

The wide variation in study methodologies made it necessary to use narrative reporting when discussing the results of this systematic review. Although the power of some of the studies was limited and the quality varied, all were considered eligible for inclusion. Moreover, they focused on different aspects of compliance in targeting $\mathrm{SpO}_{2}$. The design, $\mathrm{TR}$ of $\mathrm{SpO}_{2}$, time points and duration of each study differed.

The central finding is that compliance in targeting $\mathrm{SpO}_{2}$ was low, as were the alarm settings. All studies in compliance

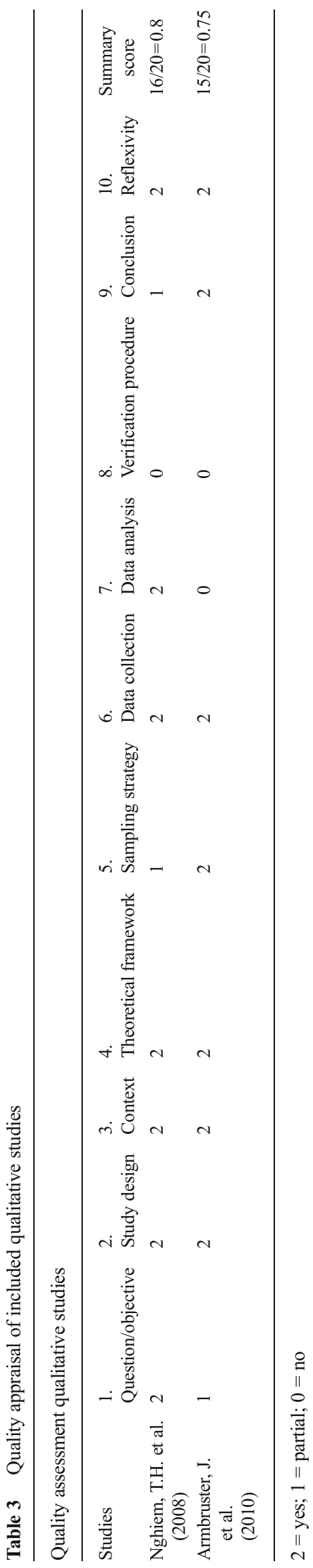




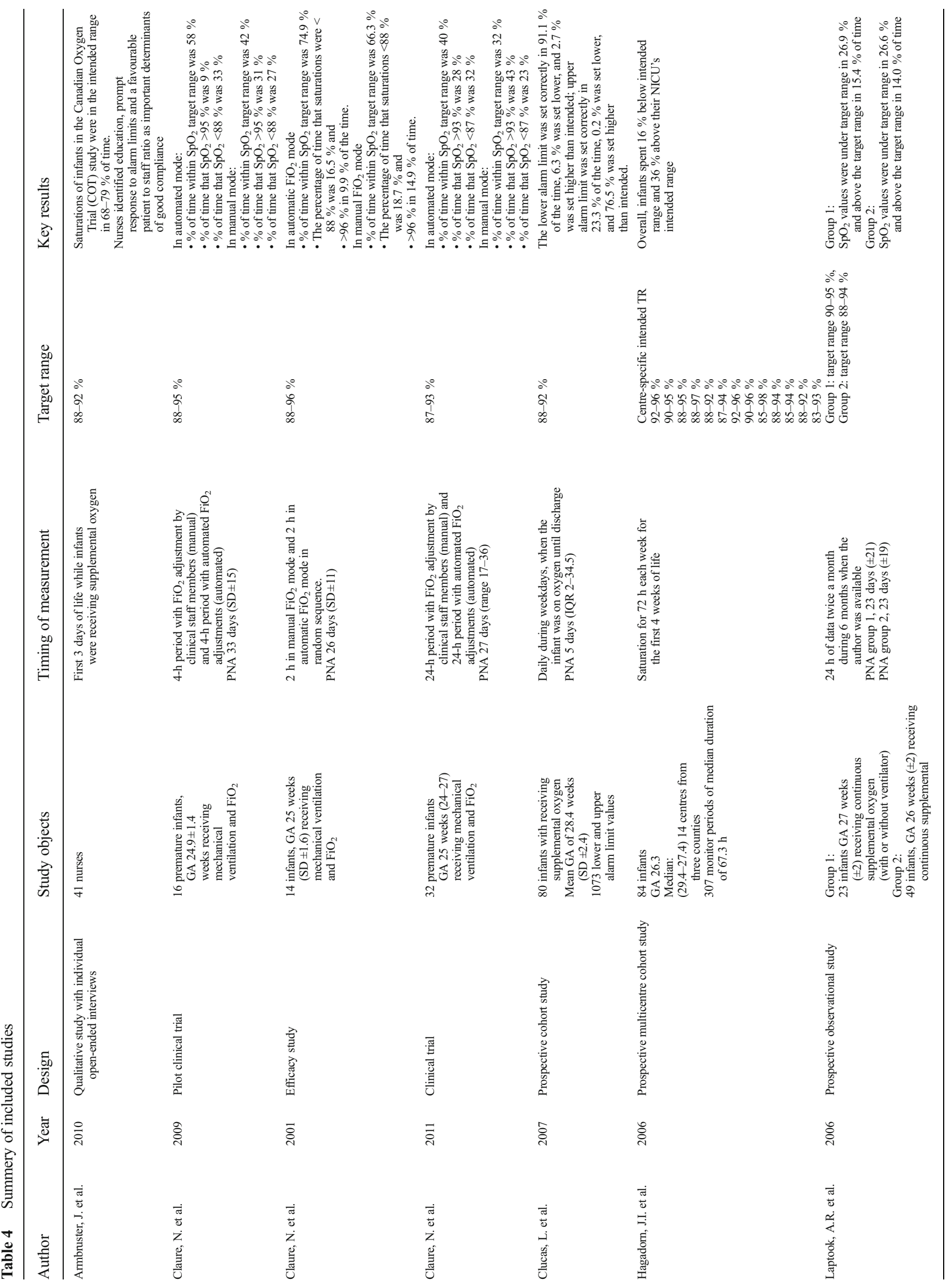




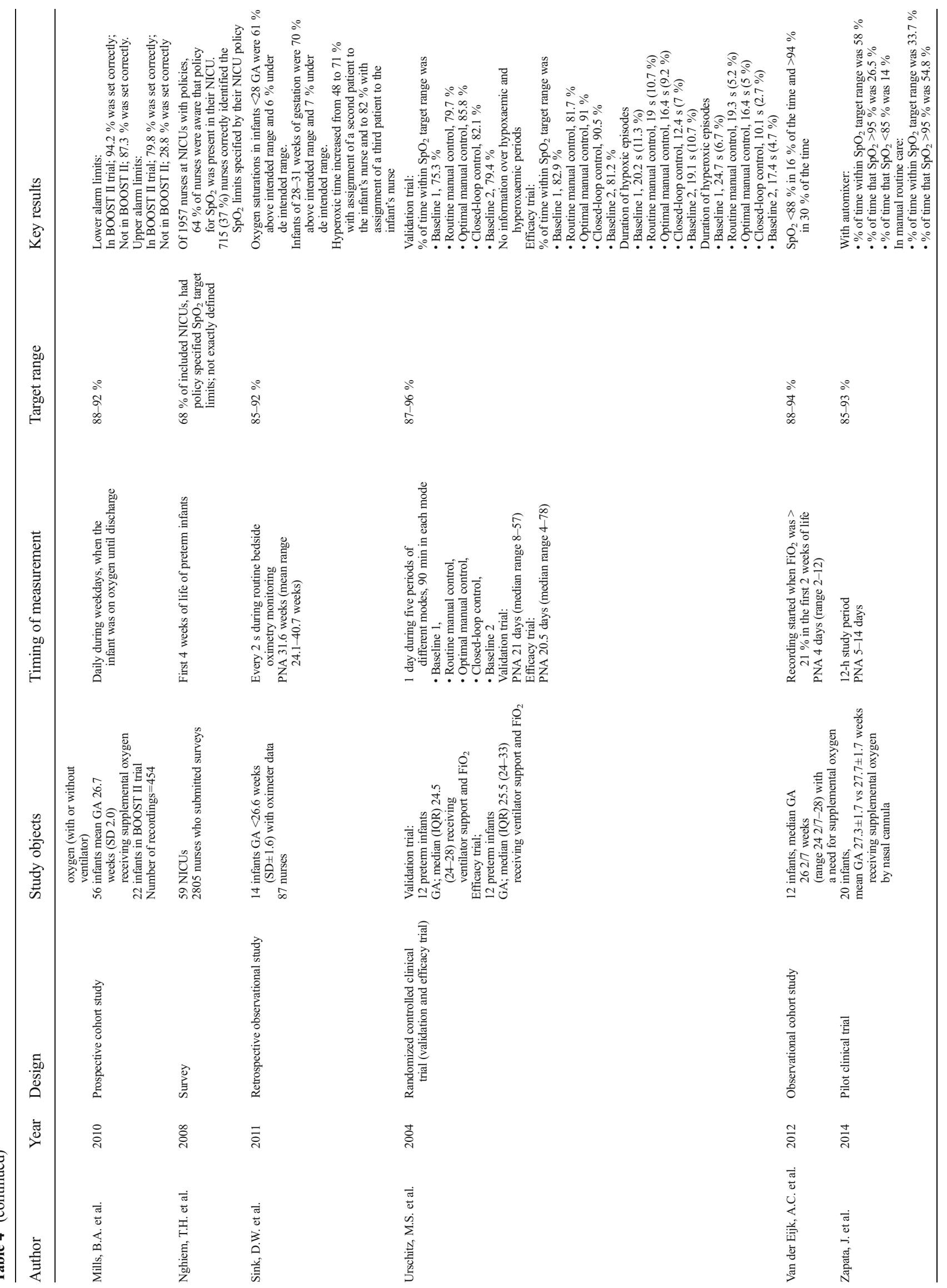




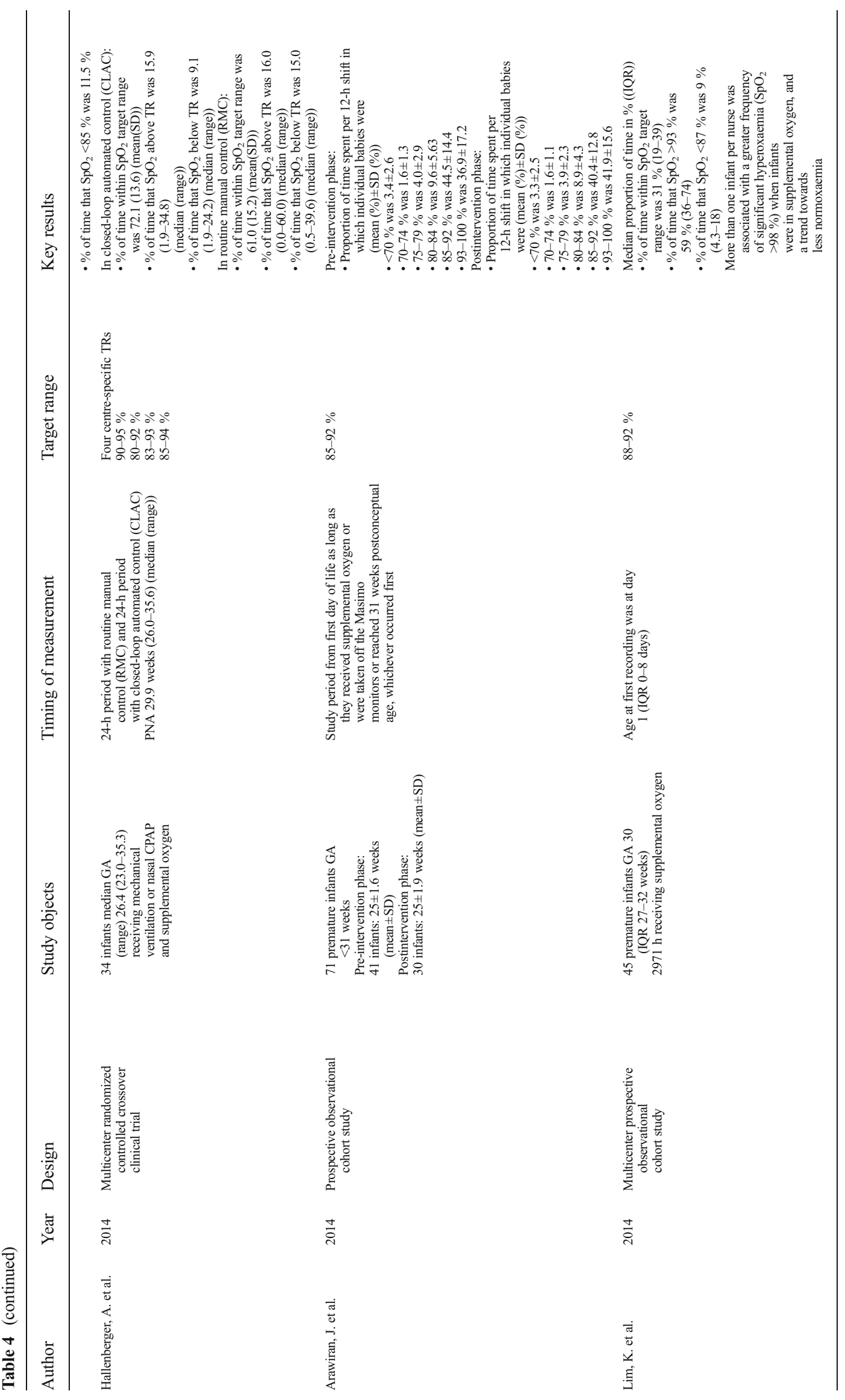


in oxygen targeting reported that maintaining the $\mathrm{SpO}_{2}$ below the upper limit was the most difficult to adhere to $[1,6,7,10$, $17,18,25,27,34,39,41]$. The analysis of the large clinical trials comparing lower- vs higher-oxygen-saturation TR was based on the intention to treat principle. However, the larger proportion of the $\mathrm{SpO}_{2}$ was either below or above the intended TR and there was also an overlap between the two TRs [4, 32, 36]. Although compliance was audited [27], it is possible that this has influenced the outcome of the trials. This underlines the importance in improving compliance in $\mathrm{SpO}_{2}$ targeting, as improved compliance could have influenced the results.

\section{According to the studies}

Several factors may play a role in low compliance in targeting oxygen saturation: lack of awareness of the TR settings, limited knowledge of the effects of hypoxaemia and hyperoxaemia and an increased nurse/patient ratio [2, 23, 25, 29, 34]. Many caregivers were unaware of the appropriate $\mathrm{SpO}_{2}$ limits [29]. In addition, nurses tend to rely on subjective observations for oxygen titration, such as skin colour and chest excursions, as well as PO and blood gases [35]. So far, studies indicate that the effects of education and training in improving the compliance targeting $\mathrm{SpO}_{2}$ are disappointing [1,23].

On the other hand, the use of automated $\mathrm{FiO} 2$ regulation, which eliminates the need for the nurses' compliance, has been shown to improve the time that $\mathrm{SpO}_{2}$ remains within TR [7-9, 38, 41]. The increase in time within TR was small, but it is possible that the effect of automated $\mathrm{FiO}_{2}$ regulation has been underestimated. A Hawthorne effect could have increased the nurses' compliance during the short study period, thus decreasing the difference between the manual and automated periods. The effectiveness of automated regulation on oxygenation variability, and whether this results in an improved outcome, remains to be investigated [5].

It has been suggested that the absence of a $\mathrm{FiO}_{2}$ titration protocol would lead to saturations which would frequently exceed or fall below the TR [24]. Manual adjustments of $\mathrm{FiO}_{2}$ can vary widely in frequency and step size, so standardization of these adjustments could decrease large fluctuations in $\mathrm{SpO}_{2}$ [39]. After implementing an oxygen titration protocol for reducing the incidence of severe ROP, Lau et al. observed that the period during which $\mathrm{SpO}_{2}$ was above TR decreased significantly [24].

Although fewer studies investigated this, compliance with alarm settings appeared to be low as well, especially the upper alarm limit [10, 27]. In addition, even when alarm limits are appropriately set, caregivers seem to have a preference for $\mathrm{SpO}_{2}$ close to the upper alarm limit $[4,20]$. This was also demonstrated in the large trials comparing TR of $\mathrm{SpO}_{2}$ [36]. It is possible that caregivers are more accustomed to preventing hypoxaemia than hyperoxaemia. It is also possible that infants are more stable in $\mathrm{SpO}_{2}$ when kept at the higher end of the TR. A regular check of alarm limit settings each shift could increase awareness of this issue.

Educational programs on hyperoxaemia improved knowledge levels $[11,16]$ but did not lead to better compliance. Earlier research has shown that after education in risks related to hyperoxaemia, the nurses' performance was still variable and only $51 \%$ of nurses were successful in minimizing exposure of their infants to hyperoxaemia [37]. Nurses usually take care of more than one patient and perform multitasking [14], and an increased workload decreases their compliance in TR $[25,34]$. Also, nurses frequently have to deal with alarms, but a large proportion of the alarms are false [33]. The common occurrence of false alarms or "cry wolf" phenomenon could lead to no or delayed response of caregivers.

The decision not to limit inclusion criteria in terms of study design and methodology led to a high level of variety within the chosen studies, necessitating a narrative review. The advantage of this method, however, is that it enabled us to have a complete overview of a range of different aspects related to compliance in $\mathrm{SpO}_{2}$ targeting. However, the review was restricted to recent studies published in English and Dutch; similar studies published in other languages may have been missed. In addition, the selection process was conducted by the first author only and selection bias could have occurred. However, in any case of doubt of including a publication, peers were approachable for discussion and were resolved by consensus to avoid bias in the selection process.

In conclusion, the main finding of this literature review is that there is a low compliance in $\mathrm{SpO}_{2}$ targeting and alarm settings during oxygen therapy in preterm infants, especially in maintaining the $\mathrm{SpO}_{2}$ below the upper limit and in setting the upper alarm limit. Although there is little data available, it is likely that training, titration protocols and decreasing the nurses' workload could improve awareness and compliance. Automated oxygen regulations have been shown to increase the time that $\mathrm{SpO}_{2}$ remains within the TR. Improving the compliance in $\mathrm{SpO}_{2}$ targeting and automated control has the potential to improve the outcome in preterm infants. The effect of training, implementing protocols and automated oxygen regulators needs further investigation.

Authors' contribution Mrs Henriëtte van Zanten was the executive researcher of the study. She performed literature search, graded the selected studies, wrote and submitted the report. Mrs Ratna Tan was involved in grading the selected studies. Mrs Agnes van den Hoogen was involved in grading the selected studies. Mr Enrico Lopriore was involved in editing of the manuscript. Mr Arjan te Pas was involved in writing and editing of the manuscript.

\section{Compliance with ethical standards}

Conflict of interest All authors declare that they have no conflict of interest to disclose. 
Ethical approval This article does not contain any studies with human participants performed by any of the authors, as this is a systematic review wherefore no ethical approval is required.

Informed consent No informed consent was obtained, as this is a systematic review wherefore no informed consent is required.

Open Access This article is distributed under the terms of the Creative Commons Attribution 4.0 International License (http:// creativecommons.org/licenses/by/4.0/), which permits unrestricted use, distribution, and reproduction in any medium, provided you give appropriate credit to the original author(s) and the source, provide a link to the Creative Commons license, and indicate if changes were made.

\section{References}

1. Arawiran J, Curry J, Welde L, Alpan G (2014) Sojourn in excessively high oxygen saturation ranges in individual, very lowbirthweight neonates. Acta Paediatr (Oslo, Norway: 1992). doi: 10.1111/apa.12827

2. Armbruster J, Schmidt B, Poets CF, Bassler D (2010) Nurses compliance with alarm limits for pulse oximetry: qualitative study. $\mathrm{J}$ Perinatol 30:531-534. doi:10.1038/jp.2009.189

3. Askie LM, Brocklehurst P, Darlow BA, Finer N, Schmidt B, Tarnow-Mordi W (2011) NeOProM: neonatal oxygenation prospective meta-analysis collaboration study protocol. BMC Pediatr 11:6. doi:10.1186/1471-2431-11-6

4. Carlo WA, Finer NN, Walsh MC, Rich W, Gantz MG, Laptook AR, Yoder BA, Faix RG, Das A, Poole WK, Schibler K, Newman NS, Ambalavanan N, Frantz ID 3rd, Piazza AJ, Sanchez PJ, Morris BH, Laroia N, Phelps DL, Poindexter BB, Cotten CM, Van Meurs KP, Duara S, Narendran V, Sood BG, O'Shea TM, Bell EF, Ehrenkranz RA, Watterberg KL, Higgins RD (2010) Target ranges of oxygen saturation in extremely preterm infants. N Engl J Med 362:19591969. doi:10.1056/NEJMoa0911781

5. Claure N (2007) Automated regulation of inspired oxygen in preterm infants: oxygenation stability and clinician workload. Anesth Analg 105:S37-41. doi:10.1213/01.ane.0000268714.51303.a5

6. Claure N, Bancalari E (2009) Automated respiratory support in newborn infants. Semin Fetal Neonatal Med 14:35-41

7. Claure N, Bancalari E, D'Ugard C, Nelin L, Stein M, Ramanathan R, Hernandez R, Donn SM, Becker M, Bachman T (2011) Multicenter crossover study of automated control of inspired oxygen in ventilated preterm infants. Pediatrics 127:E76-E83

8. Claure N, D'Ugard C, Bancalari E (2009) Automated adjustment of inspired oxygen in preterm infants with frequent fluctuations in oxygenation: a pilot clinical trial. J Pediatr 155(640-645):e641642. doi:10.1016/j.jpeds.2009.04.057

9. Claure N, Gerhardt T, Everett R, Musante G, Herrera C, Bancalari E (2001) Closed-loop controlled inspired oxygen concentration for mechanically ventilated very low birth weight infants with frequent episodes of hypoxemia. Pediatrics 107:1120-1124

10. Clucas L, Doyle LW, Dawson J, Donath S, Davis PG (2007) Compliance with alarm limits for pulse oximetry in very preterm infants. Pediatrics 119:1056-1060. doi:10.1542/peds.2006-3099

11. Deuber C, Abbasi S, Schwoebel A, Terhaar M (2013) The toxigen initiative: targeting oxygen saturation to avoid sequelae in very preterm infants. Adv Neonatal Care: Off J Natl Assoc Neonatal Nurses 13:139-145. doi:10.1097/ANC.0b013e31828913cc

12. Di Fiore JM, Bloom JN, Orge F, Schutt A, Schluchter M, Cheruvu VK, Walsh M, Finer N, Martin RJ (2010) A higher incidence of intermittent hypoxemic episodes is associated with severe retinopathy of prematurity. J Pediatr 157:69-73

13. Dracup KA, Meleis AI (1982) Compliance: an interactionist approach. Nurs Res 31:31-36

14. Edworthy J, Meredith C, Hellier E, Rose D (2013) Learning medical alarms whilst performing other tasks. Ergonomics 56:1400 1417. doi: $10.1080 / 00140139.2013 .819448$

15. Finer N, Leone $\mathrm{T}$ (2009) Oxygen saturation monitoring for the preterm infant: the evidence basis for current practice. Pediatr Res 65:375-380. doi:10.1203/PDR.0b013e318199386a

16. Ford SP, Leick-Rude MK, Meinert KA, Anderson B, Sheehan MB, Haney BM, Leeks SR, Simon SD, Jackson JK (2006) Overcoming barriers to oxygen saturation targeting. Pediatrics 118(Suppl 2): S177-186. doi:10.1542/peds.2006-0913P

17. Hagadorn JI, Furey AM, Nghiem TH, Schmid CH, Phelps DL, Pillers DA, Cole CH (2006) Achieved versus intended pulse oximeter saturation in infants born less than 28 weeks' gestation: the AVIOx study. Pediatrics 118:1574-1582. doi:10.1542/peds.20050413

18. Hallenberger A, Poets CF, Horn W, Seyfang A, Urschitz MS (2014) Closed-loop automatic oxygen control (CLAC) in preterm infants: a randomized controlled trial. Pediatrics 133:e379-385. doi:10. 1542/peds.2013-1834

19. Hummler H, Fuchs H, Schmid M (2014) Automated adjustments of inspired fraction of oxygen to avoid hypoxemia and hyperoxemia in neonates - a systematic review on clinical studies. Klin Padiatr 226: 204-210. doi:10.1055/s-0034-1375617

20. Kaufman DA, Zanelli SA, Gurka MJ, Davis M, Richards CP, Walsh BK (2014) Time outside targeted oxygen saturation range and retinopathy of prematurity. Early Hum Dev 90(Suppl 2):S35-40. doi: $10.1016 / \mathrm{s} 0378-3782(14) 50010-2$

21. Kmet LM (2004) Standard quality assessment criteria for evaluating primary research papers from a variety of fields. vol HTA \#13. Alberta Heritage Foundation for Medical Research (AHFMR), Edmonton

22. Laptook AR, Salhab W, Allen J, Saha S, Walsh M (2006) Pulse oximetry in very low birth weight infants: can oxygen saturation be maintained in the desired range? J Perinatol: Off $\mathrm{J}$ Calif Perinat Assoc 26:337-341. doi:10.1038/sj.jp.7211500

23. Laptook AR, Salhab W, Allen J, Saha S, Walsh M (2006) Pulse oximetry in very low birth weight infants: can oxygen saturation be maintained in the desired range? J Perinatol 26:337-341

24. Lau YY, Tay YY, Shah VA, Chang P, Loh KT (2011) Maintaining optimal oxygen saturation in premature infants. Permanente J 15: e108-113

25. Lim K, Wheeler KI, Gale TJ, Jackson HD, Kihlstrand JF, Sand C, Dawson JA, Dargaville PA (2014) Oxygen saturation targeting in preterm infants receiving continuous positive airway pressure. $\mathrm{J}$ Pediatr 164:730-736.e731. doi:10.1016/j.jpeds.2013.11.072

26. Martin RJ, Wang K, Koroglu O, Di Fiore J, Kc P (2011) Intermittent hypoxic episodes in preterm infants: do they matter? Neonatology 100:303-310

27. Mills BA, Davis PG, Donath SM, Clucas LM, Doyle LW (2010) Improving compliance with pulse oximetry alarm limits for very preterm infants? J Paediatr Child Health 46:255-258. doi:10.1111/j. 1440-1754.2009.01680.x

28. Moher D, Liberati A, Tetzlaff J, Altman DG (2009) Preferred reporting items for systematic reviews and meta-analyses: the PRISMA statement. J Clin Epidemiol 62:1006-1012. doi:10. 1016/j.jclinepi.2009.06.005

29. Nghiem TH, Hagadorn JI, Terrin N, Syke S, MacKinnon B, Cole $\mathrm{CH}$ (2008) Nurse opinions and pulse oximeter saturation target limits for preterm infants. Pediatrics 121:e1039-1046. doi:10. 1542/peds.2007-2257

30. Poets CF, Roberts RS, Schmidt B, Whyte RK, Asztalos EV, Bader D, Bairam A, Moddemann D, Peliowski A, Rabi Y, Solimano A, 
Nelson H (2015) Association between intermittent hypoxemia or bradycardia and late death or disability in extremely preterm infants. JAMA 314:595-603. doi:10.1001/jama.2015.8841

31. Saugstad OD, Aune D (2011) In search of the optimal oxygen saturation for extremely low birth weight infants: a systematic review and meta-analysis. Neonatology 100:1-8. doi:10.1159/ 000322001

32. Schmidt B, Whyte RK, Asztalos EV, Moddemann D, Poets C, Rabi Y, Solimano A, Roberts RS (2013) Effects of targeting higher vs lower arterial oxygen saturations on death or disability in extremely preterm infants: a randomized clinical trial. JAMA 309:2111-2120

33. Siebig S, Kuhls S, Imhoff M, Gather U, Scholmerich J, Wrede CE (2010) Intensive care unit alarms - how many do we need? Crit Care Med 38:451-456. doi:10.1097/CCM.0b013e3181cb0888

34. Sink DW, Hope SA, Hagadorn JI (2011) Nurse:patient ratio and achievement of oxygen saturation goals in premature infants. Arch Dis Child Fetal Neonatal Ed 96:F93-98. doi:10.1136/adc.2009. 178616

35. Solberg MT, Hansen TW, Bjork IT (2011) Nursing assessment during oxygen administration in ventilated preterm infants. Acta Paediatr (Oslo, Norway: 1992) 100:193-197. doi:10.1111/j.16512227.2010.02094.x

36. Stenson BJ, Tarnow-Mordi WO, Darlow BA, Simes J, Juszczak E, Askie L, Battin M, Bowler U, Broadbent R, Cairns P, Davis PG, Deshpande S, Donoghoe M, Doyle L, Fleck BW, Ghadge A, Hague W, Halliday HL, Hewson M, King A, Kirby A, Marlow N, Meyer
M, Morley C, Simmer K, Tin W, Wardle SP, Brocklehurst P (2013) Oxygen saturation and outcomes in preterm infants. N Engl J Med 368:2094-2104. doi:10.1056/NEJMoa1302298

37. Sun SC, Stefen E, Vangvanichyakorn K (2004) Validation of prescribed target $\mathrm{SpO}(2)$ range in ELBW infants: a reality check of nurses' practice. Pediatr Res 55:528A-528A

38. Urschitz MS, Horn W, Seyfang A, Hallenberger A, Herberts T, Miksch S, Popow C, Muller-Hansen I, Poets CF (2004) Automatic control of the inspired oxygen fraction in preterm infants: a randomized crossover trial. Am J Respir Crit Care Med 170:1095-1100. doi:10.1164/rccm.200407-929OC

39. van der Eijk AC, Dankelman J, Schutte S, Simonsz HJ, Smit BJ (2012) An observational study to quantify manual adjustments of the inspired oxygen fraction in extremely low birth weight infants. Acta Paediatr (Oslo, Norway: 1992) 101:e97-e104. doi:10.1111/j. 1651-2227.2011.02506.x

40. van Zanten HA, Tan RN, Thio M, de Man-van Ginkel JM, van Zwet EW, Lopriore E, Te Pas AB (2014) The risk for hyperoxaemia after apnoea, bradycardia and hypoxaemia in preterm infants. Arch Dis Child Fetal Neonatal Ed. doi:10.1136/archdischild-2013305745

41. Zapata J, Gomez JJ, Araque Campo R, Matiz Rubio A, Sola A (2014) A randomised controlled trial of an automated oxygen delivery algorithm for preterm neonates receiving supplemental oxygen without mechanical ventilation. Acta Paediatr (Oslo, Norway: 1992) 103:928-933. doi:10.1111/apa.12684 